\title{
Adaptabilidade e estabilidade de genótipos de feijoeiro-comum com grãos tipo carioca na Região Central do Brasil
}

\author{
Helton Santos Pereira ${ }^{(1)}$, Leonardo Cunha Melo(1), Luís Cláudio de Faria(1), Maria José Del Peloso(1), \\ Joaquim Geraldo Cáprio da Costa(1), Carlos Agustín Rava ${ }^{(1)}$ e Adriane Wendland(1) \\ (1)Embrapa Arroz e Feijão, Caixa Postal 179, CEP 75375-000 Santo Antônio de Goiás, GO. E-mail: helton@cnpaf.embrapa.br, \\ leonardo@cnpaf.embrapa.br, Icfaria@cnpaf.embrapa.br, mjpeloso@cnpaf.embrapa.br, caprio@cnpaf.embrapa.br, adrianew@cnpaf.embrapa.br
}

\begin{abstract}
Resumo - O objetivo deste trabalho foi identificar genótipos de feijoeiro-comum (Phaseolus vulgaris) tipo carioca, com alta adaptabilidade e estabilidade de produção, na Região Central do Brasil, pelo uso de diferentes métodos. Foram conduzidos 45 ensaios de valor de cultivo e uso, com 16 genótipos, em blocos completos ao acaso, com três repetições, nos estados de Goiás, Distrito Federal, Mato Grosso, Mato Grosso do Sul e Tocantins, nos anos de 2003 e 2004. Os dados de produtividade de grãos foram submetidos a análises de variância e de estabilidade e adaptabilidade pelos métodos de Lin \& Binns, Annichiarico, Eberhart \& Russell, Cruz et al., e AMMI (modelo de efeitos principais aditivos e interação multiplicativa). O método de Lin \& Binns, modificado por Carneiro (trapézio quadrático ponderado pelo coeficiente de variação), e o de Annichiarico mostraram-se muito úteis ao programa de melhoramento genético, pois reúnem características como: simplicidade de utilização; separação dos ambientes em favoráveis e desfavoráveis; e identificação dos genótipos mais estáveis, entre os mais produtivos. A cultivar de feijão carioca BRS Estilo reúne alta adaptabilidade, estabilidade e produtividade de grãos na Região Central do Brasil. A cultivar Pérola, amplamente utilizada no País, também apresenta boa estabilidade e adaptabilidade.
\end{abstract}

Termos para indexação: Phaseolus vulgaris, interação genótipos x ambientes, produtividade de grãos.

\section{Adaptability and stability of common bean genotypes with carioca grain type for central Brazil}

\begin{abstract}
The aim of this work was to test different methods to identify carioca grain type common bean (Phaseolus vulgaris) genotypes with high adaptability and yield stability for central Brazil. Forty-five production trials were carried out with 16 genotypes, in randomized complete block designs with three replications, in the states Goiás, Mato Grosso, Mato Grosso do Sul, Tocantins, and the Distrito Federal, in 2003 and 2004. The grain yield data were submitted to analysis of variance and to analysis of stability and adaptability using the methods of Lin \& Binns (modified by Carneiro), Annichiarico, Eberhart \& Russell, Cruz et al., and AMMI. The Lin \& Binns (modified by Carneiro) and Annichiarico's methods were very useful to the common beans genetic breeding program, because of their easiness of use in separating favorable and unfavorable environments and their identification of stable genotypes among the most productive ones. The cultivar BRS Estilo combined high adaptability, stability, and grain yield in central Brazil. The cultivar Perola, widely utilized in Brazil, also had good stability and adaptability in this region.
\end{abstract}

Index terms: Phaseolus vulgaris, genotype x environments interaction, grain yield.

\section{Introdução}

Nos últimos anos, o Brasil tem ocupado o primeiro lugar na produção e consumo mundial de feijão, Phaseolus vulgaris L. (FAO, 2007), que é um dos alimentos básicos e uma das principais fontes de proteína na alimentação da população brasileira. Entre os diversos tipos de grãos do feijoeiro-comum, merece destaque o tipo carioca, que representa $70 \%$ do mercado consumidor brasileiro (Del Peloso \&
Melo, 2005). O feijoeiro-comum é cultivado em quase todos os estados brasileiros, em diferentes sistemas de cultivo e épocas de semeadura e, portanto, a cultura está submetida às mais diversas condições ambientais.

Os programas de melhoramento têm abastecido o mercado brasileiro com novas cultivares com características desejáveis, como a estabilidade de produção, e colaborado para o aumento da produtividade média de $749 \mathrm{~kg} \mathrm{ha}^{-1}$ em 1997, para $1.044 \mathrm{~kg} \mathrm{ha}^{-1} \mathrm{em}$ 2006 (IBGE, 2008). O programa de melhoramento do 
feijoeiro-comum da Embrapa Arroz e Feijão sistematiza as avaliações das linhagens desenvolvidas em uma rede nacional organizada, que inclui a Região Central do País (Goiás, Distrito Federal, Mato Grosso, Mato Grosso do Sul e Tocantins), responsável por 14,3\% da produção nacional de feijão (IBGE, 2008). A avaliação final das linhagens é realizada nos ensaios de valor de cultivo e uso, em um grande número de ambientes, que representam as diversas condições ambientais às quais uma cultivar pode ser submetida.

Em condições de grande variação ambiental, é esperada acentuada interação genótipos $\mathrm{x}$ ambientes (GxA) (Allard \& Bradshaw, 1964), que é a resposta diferencial dos genótipos nos diferentes ambientes, especialmente quanto ao caráter produtividade de grãos. Isto é comprovado em vários trabalhos conduzidos com essa cultura no Brasil (Ramalho et al., 1998; Melo et al., 2007). O estudo dessa interação tem várias implicações em um programa de melhoramento e, na etapa de avaliação de linhagens para indicação de novas cultivares, sua importância é mais evidente e bastante pronunciada nas condições de cultivo do feijoeiro-comum. Assim, devem-se buscar alternativas para amenizar o seu efeito, com destaque para a identificação de cultivares de comportamento previsível e responsivas à melhoria do ambiente, por meio de métodos de análise de estabilidade e adaptabilidade que forneçam informações detalhadas sobre o comportamento das cultivares (Cruz \& Regazzi, 2001).

Inúmeros métodos de análise de estabilidade e adaptabilidade, baseados em diferentes princípios, já foram descritos, e os mais utilizados são baseados em regressões, com utilização de um (Eberhart \& Russel, 1966) ou dois segmentos de reta (Cruz et al., 1989). Como nem todos os dados se ajustam a modelos lineares, outra possibilidade é a utilização de métodos não paramétricos (Cruz \& Carneiro, 2003), como os de Lin \& Binns (1988), modificado por Carneiro (1998), o de Annicchiarico (1992) e o da análise da interação multiplicativa dos efeitos principais aditivos (AMMI).

O método de Lin \& Binns (1988), modificado por Carneiro (1998), identifica os genótipos mais estáveis, por meio de um único parâmetro de estabilidade e adaptabilidade, e contempla os desvios em relação à produtividade máxima obtida em cada ambiente, além de possibilitar o detalhamento dessa informação para ambientes favoráveis e desfavoráveis. O método de
Annicchiarico (1992) avalia a estabilidade por meio do risco associado em relação à adoção das cultivares. Um método mais recente de estudo de estabilidade e adaptabilidade é o AMMI, que utiliza componentes principais e estuda a interação em um modelo multiplicativo (Gauch \& Zobel, 1996).

Estudos de estabilidade e adaptabilidade têm sido realizados por meio de diversos métodos, em espécies de importância econômica, inclusive com o feijoeirocomum (Carbonell et al., 2004; Oliveira et al., 2006), e têm auxiliado na indicação de cultivares com alta capacidade produtiva, estabilidade e adaptabilidade. Contudo, estudos dessa natureza com o feijoeirocomum, na Região Central do Brasil, são escassos.

O objetivo deste trabalho foi identificar genótipos de feijoeiro-comum, com grãos carioca e alta adaptabilidade e estabilidade de produção, na Região Central do Brasil, com uso de diferentes métodos.

\section{Material e Métodos}

Os ensaios foram conduzidos em 2003 e 2004, em 45 ambientes da Região Central do Brasil, nos estados de Goiás, Distrito Federal, Mato Grosso, Mato Grosso do Sul e Tocantins, em delineamento de blocos completos ao acaso, com três repetições, e parcelas de quatro linhas com $4 \mathrm{~m}$ de comprimento. Os dados de produtividade foram coletados nas duas linhas centrais. Cada ensaio foi constituído de 16 genótipos de feijoeirocomum de grãos carioca: 12 genótipos promissores (CNFCs 9435, 9458, 9461, 9471, 9484, 9494, 9500, 9504, 9506, 9518, CNFE 8009 e Carioca 11) e quatro testemunhas amplamente plantadas no País (Pérola, IAPAR 81, Carioca Pitoco e Magnífico). Os genótipos CNFC 9435 e CNFC 9461 foram lançados como novas cultivares: a BRS 9435 Cometa e a BRS Estilo, respectivamente.

Os dados de produtividade foram submetidos a análises de variância, tendo-se considerado o efeito de tratamentos como fixo e os demais como aleatórios. Em seguida, foi realizada a análise conjunta dos ensaios. Detectou-se que a razão entre o maior e o menor quadrado médio do resíduo foi superior a sete, indicativo de que as variâncias residuais não foram homogêneas (Pimentel-Gomes, 2000). Assim, procedeu-se ao ajuste dos graus de liberdade do erro-médio e da interação GxA, conforme o método de Cochran (1954). 
Para as análises de estabilidade, foram adotados seis métodos: Eberhart \& Russell (1966), Lin \& Binns (1988) modificado por Carneiro (1998) (original com decomposição de $\mathrm{P}_{\mathrm{i}}$ ), Lin \& Binns (1988) modificado por Carneiro (1998) (trapézio quadrático ponderado pelo coeficiente de variação), Cruz et al. (1989) e Annicchiarico (1992), por meio do aplicativo GENES (Cruz, 2001), e AMMI (Zobel et al., 1988), por meio do aplicativo Estabilidade (Ferreira, 2000).

No método de Eberhart \& Russell (1966), a adaptabilidade ou a resposta linear aos ambientes é dada pela estimativa do parâmetro $\beta_{1 \mathrm{i}}$ e pela produtividade média $\left(\beta_{0 \mathrm{i}}\right)$; e a estabilidade, pela variância dos desvios da regressão $\left(\sigma_{\delta \mathrm{i}}^{2}\right)$, conforme o seguinte modelo:

$\mathrm{Y}_{\mathrm{ij}}=\beta_{0 \mathrm{i}} \mathrm{I}_{\mathrm{j}}+\delta_{\mathrm{ij}}+\bar{\varepsilon}_{\mathrm{ij}}$

em que: $Y_{\mathrm{ij}}$ é a média de produtividade de grãos $\left(\mathrm{kg} \mathrm{ha}^{-1}\right)$ do genótipo $i$, no ambiente $\mathrm{j} ; \beta_{0 \mathrm{i}}$ é a média geral; $\beta_{1 \mathrm{i}}$ é o coeficiente de regressão linear; $\delta_{\mathrm{ij}}$ é a variância dos desvio da regressão; $\bar{\varepsilon}_{\mathrm{ij}}$ é o erro experimental médio; $\mathrm{I}_{\mathrm{j}}$ é o índice ambiental codificado $\left(\Sigma_{\mathrm{j}} \mathrm{I}_{\mathrm{j}}=0\right)$, dado por $I_{j}=\frac{1}{g} \sum_{i} Y_{i j}-\frac{1}{a g} Y$, para $g$ linhagens $e$ a ambientes.

Na regressão linear bissegmentada de Cruz et al. (1989), a resposta linear aos ambientes desfavoráveis é dada pela estimativa de $\beta_{1 \text { i }}$, e a resposta aos ambientes favoráveis por $\beta_{1 \mathrm{i}}+\beta_{2 \mathrm{i}}$. A estabilidade dos genótipos é avaliada pelas variâncias dos desvios da regressão $\left(\sigma_{\delta i}^{2}\right)$ de cada genótipo, em função das variações ambientais. Nesse método, adotou-se o seguinte modelo:

$\mathrm{Y}_{\mathrm{ij}}=\mathrm{B}_{0 \mathrm{i}}+\beta_{\mathrm{Ii}} \mathrm{I}_{\mathrm{i}}+\beta_{2 \mathrm{i}} \mathrm{T}\left(\mathrm{I}_{\mathrm{i}}\right)+\delta_{\mathrm{ij}}+\bar{\varepsilon}_{\mathrm{ij}}$ em que: $Y_{\mathrm{ij}}, \beta_{0 \mathrm{i}}, \mathrm{I}_{\mathrm{j}}$ e $\bar{\varepsilon}_{\mathrm{ij}}$ são as variáveis definidas anteriormente; e

$\mathrm{T}\left(\mathrm{I}_{\mathrm{j}}\right)=0$, se $\mathrm{I}_{\mathrm{j}}<0$

$\mathrm{T}\left(\mathrm{I}_{\mathrm{j}}\right)=\mathrm{I}_{\mathrm{j}}-\mathrm{I}_{+}$, se $\mathrm{I}_{\mathrm{j}}>0$

em que $\bar{I}_{+}$é a média dos índices $\mathrm{I}_{\mathrm{j}}$ positivos.

O método de Lin \& Binns (1988) foi modificado por Carneiro (1998) e, entre os métodos propostos por esse autor, foram utilizados o original com decomposição de $\mathrm{P}_{\mathrm{i}}$ e o do trapézio quadrático ponderado pelo coeficiente de variação residual $(\mathrm{CV})$. No método original, foi realizada a decomposição de $\mathrm{P}_{\mathrm{i}}$ nas partes relativas a ambientes favoráveis e desfavoráveis. A estimativa de $\mathrm{P}_{\mathrm{i}}$ foi dada por: $\mathrm{P}_{\mathrm{i}}=\frac{\sum_{\mathrm{j}=1}^{\mathrm{a}}\left(\mathrm{Y}_{\mathrm{ij}}-\mathrm{M}_{\mathrm{j}}\right)^{2}}{2 \mathrm{a}}$, em que: $\mathrm{P}_{\mathrm{i}}$ é a estimativa do parâmetro de estabilidade do i-ésimo genótipo; $\mathrm{Y}_{\mathrm{ij}}$ é a produtividade do i-ésimo genótipo, no j-ésimo ambiente; $\mathrm{M}_{\mathrm{j}}$ é a resposta máxima, observada entre todos os genótipo no j-ésimo ambiente; a é o número de ambientes.

Os ambientes foram classificados com base nos índices ambientais, definidos como a diferença entre a média dos genótipos avaliados em cada local e a média geral. Em ambientes favoráveis, com índices positivos, inclusive o valor $0,0 \mathrm{P}_{\text {if }}$ foi estimado por equação semelhante à anterior, tendo-se substituído o termo $2 \mathrm{a}$ por $2 \mathrm{f}$, em que f é o número de ambientes favoráveis. Da mesma forma, em ambientes desfavoráveis, cujos índices são negativos, substituiu-se o termo 2 a por $2 \mathrm{~d}$, em que d é o número de ambientes desfavoráveis.

No método ponderado pelo $\mathrm{CV}$, o desempenho de cada genótipo foi estimado pela estatística a seguir:

$P_{i}=\sum_{j=1}^{n}\left[\left(\frac{Y_{g(j+1)}+Y_{g j}}{2}\right)-\left(\frac{Y_{g(j+1)}+Y_{i j}}{2}\right)\right]^{2}\left(\bar{Y}_{. j+1)}-\bar{Y}_{. j}\right)$,

em que: $P_{i}$ é a estimativa da estatística MAEC (medida de adaptabilidade e estabilidade de comportamento) do genótipo i; $Y_{\mathrm{ij}}$ é a produtividade do i-ésimo genótipo, no j-ésimo ambiente; $Y_{\mathrm{gj}}$ é a estimativa da produtividade do genótipo hipotético ideal, no ambiente $\mathrm{j}$, conforme modelo de Cruz et al. (1989), dado por:

$Y_{g j}=\beta_{0 \mathrm{~g}}+\beta_{1 \mathrm{~g}} \mathrm{I}_{\mathrm{j}}+\beta_{2 \mathrm{~g}} \mathrm{~T}\left(\mathrm{I}_{\mathrm{j}}\right)$,

em que $\beta_{0 \mathrm{~g}}$ é a máxima produtividade encontrada em todo o ensaio; $\beta_{1 \mathrm{~g}}=0,5$ e $\beta_{2 \mathrm{~g}}=1$ são os valores estabelecidos por Cruz \& Carneiro (2003) - um reflete baixa resposta aos ambientes desfavoráveis $\left(\beta_{1 \mathrm{~g}}=0,5\right)$, e outro é responsivo às condições favoráveis $\left(\beta_{1 \mathrm{~g}}+\beta_{2 \mathrm{~g}}=1,5\right)$.

A estatística $P_{i}$ é multiplicada pelo fator $f$, definido por $\mathrm{f}=\mathrm{CV}_{\mathrm{j}} / \mathrm{CV}_{\mathrm{T}}$, em que $\mathrm{CV}_{\mathrm{j}}$ representa o coeficiente de variação no ambiente $\mathrm{j}, \mathrm{e} \mathrm{CV}_{\mathrm{T}}$, a soma dos coeficientes de variação dos $\mathrm{j}$ ambientes. Assim, linhagens com menor valor de $\mathrm{P}_{\mathrm{i}}$ apresentam comportamento mais próximo ao do genótipo hipotético ideal, e a similaridade dos locais, bem como a precisão de cada experimento, são levados em consideração.

O método de Annicchiarico (1992) é baseado no chamado índice de confiança genotípico, estimado por: $\omega_{\mathrm{i}(\mathrm{g})}=\hat{\mu}_{\mathrm{i}(\mathrm{g})}-\mathrm{z}_{(1-\alpha)} \hat{\sigma}_{\mathrm{z}_{\mathrm{i}}(\mathrm{g})}$, em que são considerados todos os ambientes e em que: $\hat{\mu}_{\mathrm{i}(\mathrm{g})}$ é a média percentual dos genótipos $\mathrm{i} ; \hat{\sigma}_{\mathrm{z}_{\mathrm{i}}(\mathrm{g})}$ é o desvio-padrão dos valores $\mathrm{z}_{\mathrm{ij}}$, associado ao i-ésimo genótipo; $\mathrm{z}_{(1-\alpha)}$ é o percentil da função de distribuição normal padrão. 
O índice foi calculado, também, para os ambientes favoráveis e desfavoráveis. O coeficiente de confiança adotado foi de $75 \%$, isto é, $\alpha=0,25$.

Para utilização do método AMMI, o modelo utilizado foi:

$Y_{i j}=\mu+g_{i}+a_{j}+\sum_{k=1}^{n} \lambda_{k} \gamma_{i k} \alpha_{j k}+\rho_{i j}+\bar{\varepsilon}_{i j}$,

em que: $Y_{i j}$ é a resposta média do genótipo $\mathrm{i}(\mathrm{i}=1,2, \ldots$, $\mathrm{G}$ genótipos) no ambiente $\mathrm{j}(\mathrm{j}=1,2, \ldots, \mathrm{A}$ ambientes); $\mu$ é a média geral dos ensaios; $g_{i}$ é o efeito fixo do genótipo $\mathrm{i} ; \mathrm{a}_{\mathrm{j}}$ é o efeito fixo do ambiente $\mathrm{j} ; \lambda_{\mathrm{k}}$ é o k-ésimo valor singular (escalar) da matriz de interações original (denotada por GA); $\gamma_{\mathrm{ik}}$ é o elemento correspondente ao i-ésimo genótipo, no k-ésimo vetor singular coluna da matriz GA; $\alpha_{\mathrm{jk}}$ é o elemento correspondente ao j-ésimo ambiente, no k-ésimo vetor singular linha da matriz GA; $\rho_{\mathrm{ij}}$ é o resíduo associado ao termo $(\mathrm{ga})_{\mathrm{ij}}$ da interação clássica do genótipo i com o ambiente $\mathrm{j}$; $\bar{\varepsilon}_{\mathrm{ij}}$ é o erro experimental médio. $\mathrm{O}$ teste $\mathrm{F}$ de Gollob foi utilizado para seleção do modelo. Para identificação dos genótipos mais estáveis e adaptados, foram utilizados os escores dos genótipos para os dois primeiros componentes principais.

\section{Resultados e Discussão}

A maioria dos ensaios apresentou efeitos significativos de genótipos, e os valores de coeficiente de variação $(\mathrm{CV})$ foram todos iguais ou inferiores a $25 \%$ (Tabela 1). Houve variação nas médias e CV dos ensaios, evidência de que as condições a que foram submetidos os genótipos foram discrepantes. Na análise conjunta, todos os efeitos foram significativos, o que é indicativo da presença de variabilidade para seleção entre os genótipos, variabilidade entre os ambientes utilizados e a ocorrência de resposta diferencial dos genótipos aos ambientes, o que determina a necessidade de análises de estabilidade (Tabela 2).

Para Eberhart \& Russell (1966), a adaptabilidade do genótipo é dada pelo parâmetro $\beta_{1 \mathrm{i}}$, e a estabilidade de comportamento é medida por meio da variância dos desvios da regressão $\sigma_{\mathrm{di}}^{2}$ e pelo coeficiente de determinação $\left(\mathrm{R}^{2}\right)$ que, segundo Cruz \& Regazzi (2001), é uma medida auxiliar na avaliação da estabilidade, quando as $\sigma_{\mathrm{di}}^{2}$ são significativas. As cultivares BRS Estilo, Pérola e IAPAR 81 mostraram-se adaptadas a ambientes favoráveis $\left(\hat{\beta}_{1 \mathrm{i}}>1\right)$ (Tabela 3 ).

Com relação à estabilidade de comportamento, as $\sigma_{\mathrm{di}}^{2}$ foram significativas e o $\mathrm{R}^{2}$ foi superior a $80 \%$, o que indica que a previsibilidade dessas cultivares é aceitável. Entre essas cultivares, merecem destaque BRS Estilo e Pérola, que apresentaram alta média e estiveram presentes no grupo de maior produtividade. Somente o genótipo CNFC 9484 foi identificado como adaptado a ambientes desfavoráveis $\left(\hat{\beta}_{1 \mathrm{i}}<1\right)$, com previsibilidade aceitável, porém com produtividade abaixo da média. Os demais genótipos apresentaram adaptabilidade geral $\left(\hat{\beta}_{1 \mathrm{i}}=1\right)$, e merece destaque o CNFC 9518, que mostrou previsibilidade tolerável e figurou no grupo de maior produtividade, pelo teste de médias.

Segundo Cruz et al. (1989), o genótipo de comportamento ideal possui elevada média produtiva, baixa sensibilidade a ambientes desfavoráveis $\left(\hat{\beta}_{1 \mathrm{i}}<1\right)$, responsividade a melhoria ambiental $\left(\hat{\beta}_{1 \mathrm{i}}+\hat{\beta}_{2 \mathrm{i}}>1\right) \mathrm{e}$ previsibilidade alta ( $\sigma_{\mathrm{di}}^{2}$ não-significativas) ou tolerável, $\left(\sigma_{\mathrm{di}}^{2}\right.$ significativas e $\mathrm{R}^{2}$ acima de $80 \%$ ). Nenhum dos genótipos avaliados apresentou o comportamento ideal (Tabela 3), o que evidencia uma limitação desse método, conforme comentado por Oliveira et al. (2006) e Albrecht et al. (2007), em estudos com espécies diferentes.

O genótipo CNFE 8009, identificado como de adaptação ampla por Eberhart \& Russell (1966), mostrou-se pouco responsivo às melhorias ambientais $\left(\hat{\beta}_{1 \mathrm{i}}+\hat{\beta}_{2 \mathrm{i}}<1\right)$. Além disso, os genótipos CNFC 9484 e CNFC 9500, de adaptação ampla por Eberhart \& Russell (1966), apresentaram baixa sensibilidade a ambientes desfavoráveis $\left(\hat{\beta}_{1 \mathrm{i}}<1\right)$ por Cruz et al. (1989).

Entre os genótipos de maior produtividade, BRS Estilo e Pérola mostraram-se sensíveis a ambientes desfavoráveis, responsivos à melhoria do ambiente e com previsibilidade tolerável. O genótipo CNFC 9518 apresentou sensibilidade a ambientes desfavoráveis, não responsividade à melhoria ambiental e previsibilidade tolerável. Assim, o método de Cruz et al. (1989) trouxe um maior refinamento das informações sobre os genótipos, em comparação ao de Eberhart \& Russell (1966). Entretanto, ao se considerar o grande número de parâmetros envolvidos, a identificação das linhagens que mais se aproximaram do ideal ou adaptadas a ambientes específicos ficou comprometida pelo método de Cruz et al. (1989), fato já relatado por Oliveira et al. (2006).

Segundo o método de Lin \& Binns (1988), modificado por Carneiro (1998), o genótipo mais estável apresenta o menor desvio em relação à produtividade máxima de 
cada ambiente, ou seja, menor valor de $\mathrm{P}_{\mathrm{i}}$. No método que utiliza somente a decomposição de $\mathrm{P}_{\mathrm{i}}$, os genótipos mais estáveis/adaptados, quando são considerados todos os ambientes, foram BRS Estilo, CNFC 9518 e Pérola (Tabela 4). O genótipo BRS Estilo foi o mais estável/adaptado aos ambientes favoráveis, seguido por IAPAR 81 e Pérola. Nos ambientes desfavoráveis, novamente merecem destaque os genótipos CNFC 9518, BRS Estilo e Pérola. Uma grande vantagem do método de Lin \& Binns,

Tabela 1. Características dos locais e resumo das análises de variância individuais dos dados de produção $\left(\mathrm{kg} \mathrm{ha}^{-1}\right)$ dos 45 ensaios de valor de cultivo e uso, conduzidos na Região Central do Brasil, nos anos de 2003 e 2004, em diferentes épocas de semeadura ${ }^{(1)}$.

\begin{tabular}{|c|c|c|c|c|c|c|}
\hline Época & Local & Altitude (m) & QMg & QMe & Média & $\mathrm{CV}$ \\
\hline & \multicolumn{6}{|c|}{2003} \\
\hline \multirow[t]{6}{*}{ Seca } & Santo Antônio de Goiás, GO & 823 & $190.166^{* *}$ & 63.884 & 1.275 & 20 \\
\hline & Panamá, GO & 733 & $199.082 * *$ & 49.765 & 1.361 & 16 \\
\hline & Dourados, MS & 430 & $166.630 *$ & 72.568 & 1.790 & 15 \\
\hline & Glória de Dourados, MS & 400 & $140.380^{*}$ & 57.563 & 1.426 & 17 \\
\hline & Aquidauana, MS & 147 & $164.604 * *$ & 26.227 & 1.699 & 10 \\
\hline & Sinop, MT & 345 & $97.358^{\mathrm{ns}}$ & 65.628 & 1.870 & 14 \\
\hline \multirow[t]{11}{*}{ Inverno } & Planaltina, DF & 1.000 & $200.893 *$ & 91.425 & 2.359 & 13 \\
\hline & Goiatuba, GO & 447 & $293.481 * *$ & 53.005 & 1.685 & 14 \\
\hline & Santo Antônio de Goiás, GO & 823 & $461.629 * *$ & 86.877 & 1.827 & 16 \\
\hline & Rio Verde, GO & 715 & $494.769 * *$ & 117.006 & 2.710 & 13 \\
\hline & Urutaí, GO & 900 & $692.971 * *$ & 95.784 & 2.452 & 13 \\
\hline & Cristalina, GO & 1.239 & $1.643 .743 * *$ & 569.989 & 3.479 & 22 \\
\hline & Cáceres, MT & 118 & $116.032^{\mathrm{ns}}$ & 153.598 & 2.478 & 16 \\
\hline & Dueré, TO & 235 & $372.595 * *$ & 61.740 & 1.471 & 17 \\
\hline & Dianópolis, TO & 693 & $388.181 *$ & 154.771 & 3.347 & 12 \\
\hline & Palmas, TO & 230 & $110.650^{\mathrm{ns}}$ & 142.905 & 2.258 & 17 \\
\hline & Araguatins, TO & 103 & $357.227 * *$ & 13.187 & 836 & 14 \\
\hline \multirow[t]{7}{*}{ Águas } & Planaltina, DF & 1.000 & $69.367^{\mathrm{ns}}$ & 189.169 & 2.106 & 21 \\
\hline & Rio Verde, GO & 715 & $332.277 * *$ & 88.291 & 1.891 & 16 \\
\hline & Ipameri, GO & 605 & $461.563 * *$ & 127.779 & 2.819 & 13 \\
\hline & Anápolis, GO & 1.018 & $350.566^{* *}$ & 69.003 & 1.369 & 19 \\
\hline & Formosa, GO & 912 & $762.515 * *$ & 81.362 & 1.483 & 19 \\
\hline & Sinop, MT & 345 & $303.296^{* *}$ & 32.605 & 973 & 19 \\
\hline & \multicolumn{6}{|c|}{2004} \\
\hline \multirow[t]{4}{*}{ Seca } & Anápolis, GO & 1.018 & $256.667 * *$ & 91.918 & 1.398 & 22 \\
\hline & Santo Antônio de Goiás, GO & 823 & $132.307^{\mathrm{ns}}$ & 109.817 & 1.840 & 18 \\
\hline & Aquidauana, MS & 147 & $246.049 * *$ & 22.022 & 1.297 & 11 \\
\hline & Sinop, MT & 345 & $188.390 * *$ & 43.079 & 1.050 & 20 \\
\hline \multirow[t]{11}{*}{ Inverno } & Planaltina, DF & 1.000 & $338.856^{\mathrm{ns}}$ & 263.357 & 3.853 & 13 \\
\hline & Anápolis, GO & 1.018 & $567.112^{\mathrm{ns}}$ & 318.029 & 2.843 & 20 \\
\hline & Santo Antônio de Goiás, GO & 823 & $191.454^{\mathrm{ns}}$ & 139.165 & 2.729 & 14 \\
\hline & Rio Verde, GO & 715 & $225.480^{*}$ & 91.255 & 3.075 & 10 \\
\hline & Itumbiara, GO & 448 & $334.232 * *$ & 111.910 & 1.835 & 18 \\
\hline & Morrinhos, GO & 771 & $194.686^{\mathrm{ns}}$ & 201.545 & 1.745 & 25 \\
\hline & Cristalina, GO & 1.239 & $1.298 .155^{* *}$ & 311.833 & 2.551 & 22 \\
\hline & Cáceres, MT & 118 & $543.376^{* *}$ & 68.437 & 2.154 & 12 \\
\hline & Dueré, TO & 235 & $289.730 * *$ & 76.873 & 1.516 & 18 \\
\hline & Gurupi, TO & 287 & $977.068 * *$ & 12.735 & 1.437 & 8 \\
\hline & Dianópolis, TO & 693 & $776.043 * *$ & 139.708 & 2.694 & 14 \\
\hline \multirow[t]{7}{*}{ Águas } & Planaltina, DF & 1.000 & $412.128^{* *}$ & 102.792 & 3.480 & 9 \\
\hline & Anápolis, GO & 1.018 & $475.057 * *$ & 107.186 & 2.307 & 14 \\
\hline & Santo Antônio de Goiás, GO & 823 & $269.968 * *$ & 22.412 & 1.091 & 14 \\
\hline & Rio Verde, GO & 715 & $1.097 .641 * *$ & 145.613 & 2.708 & 14 \\
\hline & Morrinhos, GO & 771 & $172.761 \mathrm{~ns}$ & 97.059 & 1.687 & 18 \\
\hline & Urutaí, GO & 900 & $268.452 * *$ & 29.728 & 1.755 & 10 \\
\hline & Cristalina, GO & 1.239 & $582.644 * *$ & 36.981 & 1.286 & 15 \\
\hline
\end{tabular}

${ }^{(1)} \mathrm{QMg}$ : quadrado médio de genótipos; QMe: quadrado médio do erro; $\mathrm{CV}$ : coeficiente de variação (\%); Média: média geral do ensaio (kg ha-1).

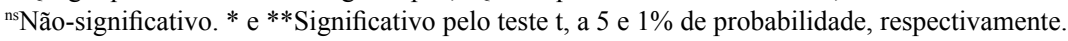


modificado por Carneiro, é a recomendação imediata de genótipos mais estáveis, em razão da unicidade do parâmetro $\mathrm{P}_{\mathrm{i}} \mathrm{e}$, ainda, uma avaliação do comportamento do genótipo em função da variação do ambiente. Além disso, os genótipos identificados como mais estáveis e adaptados geralmente figuram entre os mais produtivos.

O método do trapézio quadrático ponderado pelo $\mathrm{CV}$ identificou os genótipos BRS Estilo, Carioca

Tabela 2. Resumo da análise de variância conjunta para a produtividade de grãos $\left(\mathrm{kg} \mathrm{ha}^{-1}\right)$ e da análise AMMI de 16 genótipos de feijoeiro-comum, avaliados em 45 ensaios conduzidos na Região Central do Brasil.

\begin{tabular}{|c|c|c|c|}
\hline Fonte de variação & GL & $\begin{array}{c}\text { Soma de } \\
\text { quadrados }\end{array}$ & $\begin{array}{l}\text { Quadrado } \\
\text { médio }\end{array}$ \\
\hline Repetição/ambiente & 90 & 32.584 .590 & 362.051 \\
\hline Ambientes (A) & 44 & 1.153 .900 .264 & $26.225 .006^{* *}$ \\
\hline Genótipos (G) & 15 & 27.472 .065 & $1.831 .471 * *$ \\
\hline GxA & $(376)^{(1)}$ & 250.784 .856 & $666.981 * *$ \\
\hline $\mathrm{CP} 1$ & 58 & 44.829 .244 & $772.918 * *$ \\
\hline Resíduo 1 & 318 & 205.955 .576 & $674.659 * *$ \\
\hline $\mathrm{CP} 2$ & 56 & 38.199 .336 & $682.131 * *$ \\
\hline Resíduo 2 & 262 & 167.756 .240 & $640.290 * *$ \\
\hline CP3 & 54 & 33.125 .166 & $613.429 * *$ \\
\hline Resíduo 3 & 208 & 134.631 .074 & $647.264 * *$ \\
\hline Resíduo & $(761)^{(1)}$ & 150.227 .488 & 197.408 \\
\hline Total & 2.159 & - & - \\
\hline Média & & 2.028 & \\
\hline CV $(\%)$ & & 21,9 & \\
\hline
\end{tabular}

Pitoco e CNFC 9518 como os mais adaptados/ estáveis, quando foram considerados todos os ambientes. Os genótipos BRS Estilo, IAPAR 81 e Carioca Pitoco se destacaram em ambientes favoráveis, e CNFC 9518, BRS Estilo e Carioca Pitoco, nos ambientes desfavoráveis. As duas modificações no método de Lin \& Binns (1988) identificaram os mesmos genótipos como mais estáveis/adaptados, porém, deve-se lembrar que os ensaios utilizados não apresentaram grande variação no CV; todos foram abaixo de $25 \%$ (Tabela 1 ).

O método de Annichiarico (1992) identificou, para os genótipos BRS Estilo e CNFC 9518, índice de confiança $\left(\mathrm{W}_{\mathrm{i}}\right)$ acima de $100 \%$, o que indica que esses genótipos superam a média dos ambientes em no mínimo 5,4 e 4,2\% de produção, respectivamente (Tabela 4 ), com $75 \%$ de confiança. Nos ambientes favoráveis, novamente os genótipos que se destacaram foram CNFC 9518 e BRS Estilo, que superaram a média dos ambientes em 8,6 e $6,3 \%$ de produção, respectivamente. Nos ambientes desfavoráveis, os genótipos IAPAR 81 e BRS Estilo se destacaram com 4,8 e $4,2 \%$ de superioridade de produção, respectivamente.

$\mathrm{Na}$ análise AMMI, o modelo adequado associa significância para os eixos e não-significância para o resíduo. O modelo AMMI 3 apresentou significância dos três primeiros componentes e de seus resíduos, indicativo de que esses componentes,

Tabela 3. Estimativas de parâmetros de adaptabilidade e estabilidade fenotípica, pelos métodos de Eberhart \& Russel (1966), Cruz et al. (1989) e AMMI (Zobel et al., 1988), para 16 genótipos de feijoeiro-comum, avaliados em 45 ambientes na Região Central do Brasil, nos anos de 2003 e 2004.

\begin{tabular}{|c|c|c|c|c|c|c|c|c|c|c|c|c|}
\hline \multirow[t]{2}{*}{ Genótipo } & \multirow[t]{2}{*}{ Média $^{(1)}$} & \multicolumn{3}{|c|}{ Eberhart \& Russell } & \multicolumn{4}{|c|}{ Cruz et al. } & \multicolumn{4}{|c|}{ AMMI } \\
\hline & & $\hat{\beta}_{1 \mathrm{i}}^{(2)}$ & $\hat{\sigma}_{\mathrm{di}}{ }^{(3)}$ & $\mathrm{R}^{2}(\%)$ & $\hat{\beta}_{1 \mathrm{i}}{ }^{(2)}$ & $\hat{\beta}_{1 \mathrm{i}}+\hat{\beta}_{2 \mathrm{i}}$ & $\hat{\sigma}_{\mathrm{di}}^{(3)}$ & $\mathrm{R}^{2}(\%)$ & CP $1(18 \%)^{(5)}$ & CP $2(15 \%)^{(5)}$ & $\operatorname{MPEA}^{(6)}$ & $C^{(7)}$ \\
\hline BRS Estilo & $2.225 \mathrm{a}$ & $1,12^{*}$ & $63.560^{* *}$ & 84 & $1,08^{\mathrm{ns}}$ & $1,29 *$ & $385.949 * *$ & 85 & $-23,8$ & $-7,9$ & 16,5 & 12 \\
\hline CNFC 9518 & $2.185 \mathrm{a}$ & $0,94^{\mathrm{ns}}$ & $51.448 * *$ & 81 & $0,91^{\mathrm{ns}}$ & $1,06^{\mathrm{ns}}$ & $354.339 * *$ & 81 & 17,9 & $-5,5$ & 12,2 & 7 \\
\hline Pérola & $2.156 \mathrm{a}$ & $1,14 * *$ & $64.431 * *$ & 85 & $1,09^{\mathrm{ns}}$ & $1,34 * *$ & $384.933 * *$ & 85 & 9,0 & $-5,3$ & 7,3 & 2 \\
\hline Carioca Pitoco & $2.122 b$ & $1,02^{\mathrm{ns}}$ & $40.523 * *$ & 84 & $1,02^{\mathrm{ns}}$ & $0,99^{\mathrm{ns}}$ & $326.386^{* *}$ & 84 & $-5,6$ & 10,2 & 7,7 & 3 \\
\hline CNFE 8009 & $2.079 b$ & $0,95^{\mathrm{ns}}$ & $82.444 * *$ & 77 & $0,99^{\mathrm{ns}}$ & $0,72^{*}$ & $436.645^{* *}$ & 78 & 9,1 & $-22,2$ & 15,1 & 10 \\
\hline IAPAR 81 & $2.075 b$ & $1,15^{* *}$ & $69.115 * *$ & 85 & $1,24 * *$ & $0,73 *$ & $347.893 * *$ & 87 & $-12,7$ & 19,0 & 15,6 & 11 \\
\hline CNFC 9506 & $2.052 b$ & $0,95^{\mathrm{ns}}$ & $40.228 * *$ & 83 & $0,93^{\text {ns }}$ & $1,05^{\mathrm{ns}}$ & $322.039 * *$ & 83 & $-7,2$ & $-9,2$ & 8,1 & 4 \\
\hline CNFC 9458 & $2.044 \mathrm{~b}$ & $0,97^{\mathrm{ns}}$ & $11.901^{\mathrm{ns}}$ & 87 & $1,02^{\mathrm{ns}}$ & $0,70^{*}$ & $212.901^{\mathrm{ns}}$ & 88 & $-10,7$ & 6,7 & 8,9 & 5 \\
\hline CNFC 9471 & $2.008 \mathrm{c}$ & $1,03^{\mathrm{ns}}$ & $54.405 * *$ & 83 & $1,05^{\mathrm{ns}}$ & $0,89^{\mathrm{ns}}$ & $362.456^{* *}$ & 83 & 12,9 & 23,7 & 17,9 & 13 \\
\hline CNFC 9504 & $1.995 \mathrm{c}$ & $0,95^{\mathrm{ns}}$ & $6.593^{\mathrm{ns}}$ & 88 & $0,94^{\mathrm{ns}}$ & $1,03^{\mathrm{ns}}$ & $220.077^{\mathrm{ns}}$ & 88 & 4,6 & 9,1 & 6,7 & 1 \\
\hline CNFC 9484 & $1.992 \mathrm{c}$ & $0,89^{*}$ & $38.220 *$ & 81 & $0,84 * *$ & $1,11^{\mathrm{ns}}$ & $301.164 *$ & 82 & 21,6 & $-15,8$ & 18,9 & 14 \\
\hline Magnífico & $1.963 \mathrm{c}$ & $1,09^{\mathrm{ns}}$ & $40.095 * *$ & 86 & $1,08^{\mathrm{ns}}$ & $1,14^{\mathrm{ns}}$ & $324.363 * *$ & 86 & $-15,1$ & $-1,7$ & 8,9 & 6 \\
\hline CNFC 9500 & $1.961 \mathrm{c}$ & $0,91^{\mathrm{ns}}$ & $47.874 * *$ & 80 & $0,87 *$ & $1,11^{\mathrm{ns}}$ & $334.796^{* *}$ & 81 & 10,1 & 20,1 & 14,7 & 9 \\
\hline CNFC 9494 & $1.961 \mathrm{c}$ & $0,98^{\mathrm{ns}}$ & $8.641^{\mathrm{ns}}$ & 88 & $1,02^{\mathrm{ns}}$ & $0,82^{\mathrm{ns}}$ & $218.664^{\mathrm{ns}}$ & 88 & $-10,0$ & 15,9 & 12,7 & 8 \\
\hline BRS 9435 Cometa & $1.885 \mathrm{c}$ & $0,90^{\mathrm{ns}}$ & $68.919^{* *}$ & 77 & $0,91^{\mathrm{ns}}$ & $0,86^{\mathrm{ns}}$ & $413.190 * *$ & 77 & 26,2 & $-11,3$ & 19,3 & 15 \\
\hline Carioca 11 & $1.759 \mathrm{~d}$ & $1,03^{\mathrm{ns}}$ & $102.523 * *$ & 78 & $1,00^{\mathrm{ns}}$ & $1,15^{\mathrm{ns}}$ & $511.120 * *$ & 78 & $-26,3$ & $-26,0$ & 26,2 & 16 \\
\hline
\end{tabular}

${ }^{(1)}$ Médias seguidas por letras iguais não diferem entre si, pelo teste Scott-Knott, a $10 \%$ de probabilidade. ${ }^{(2)} \mathrm{H}_{0}: \beta_{1 \mathrm{i}}=1 .{ }^{(3)} \mathrm{H}_{0}: \sigma_{\mathrm{di}}=0 .{ }^{(4)} \mathrm{H}_{0}: \beta_{1 \mathrm{i}}+\beta_{2 \mathrm{i}}=1$. ${ }^{(5)}$ Percentagem da variação explicada pelo componente principal (CP). ${ }^{(6)}$ Média ponderada dos escores absolutos. ${ }^{(7)} \mathrm{Classificação.}{ }^{\text {ns }} \mathrm{Não}-\mathrm{significativo.}$ * e **Significativo pelo teste $\mathrm{t}$, a 5 e $1 \%$ de probabilidade, respectivamente. 
em conjunto, foram insuficientes para explicar os efeitos da interação GxA (Tabela 2) (Borges et al., 2000).

A contribuição de cada componente principal para explicar a soma de quadrados da interação foi baixa, como normalmente tem sido relatado, inclusive em outras culturas (Rocha et al., 2004, 2007). Os três primeiros, em conjunto, explicaram apenas $46 \%$ da variação $(\mathrm{CP} 1=18 \%$; $\mathrm{CP} 2=15 \%$; CP3 $=13 \%)$, abaixo dos aproximadamente $60 \%$ obtidos por Borges et al. (2000), Carbonell et al. (2004) e Melo et al. (2007), também em feijoeiro-comum. Quando os primeiros componentes principais não explicam um percentual superior a $70 \%$ da variação da interação, os resultados são insatisfatórios, e a utilização de modelos com um número maior que três componentes é, do ponto de vista biológico, insatisfatória e de difícil manipulação, em relação aos possíveis benefícios que trariam (Arias et al., 1996; Borges et al., 2000). Porém, segundo Gauch \& Zobel (1996), os primeiros componentes AMMI captam maior percentagem do desempenho real "padrão" e, com a subsequente acumulação de componentes, ocorre diminuição na percentagem de "padrão" e aumento de informações imprecisas ("ruídos"). Portanto, apesar da seleção de poucos componentes que expliquem pequena proporção da variação, a informação fornecida pela metodologia
AMMI pode ser considerada de melhor qualidade do que a fornecida por métodos tradicionais.

Para a identificação das linhagens mais estáveis por AMMI, foram utilizadas as informações dos dois primeiros componentes, para a obtenção da média dos escores absolutos de cada genótipo, ponderada pela percentagem de explicação de cada componente (Tabela 3). Quanto menor o valor da média dos escores, menor a contribuição do genótipo para a interação e, consequentemente, mais estável é o genótipo. Assim, os genótipos mais estáveis foram CNFC 9504, Pérola e Carioca Pitoco (Tabela 3 e Figura 1 A). Entre os genótipos de maior produtividade, merece destaque, mais uma vez, o Pérola, que foi o segundo mais estável (Figura $1 \mathrm{~B}$ ).

Como os parâmetros de estabilidade e adaptabilidade em cada método são diferentes, os métodos identificaram diferentes genótipos como os mais estáveis. Entre esses, pode-se destacar o BRS Estilo, uma nova cultivar de feijão carioca, indicada inicialmente para os estados de Goiás, Distrito Federal e Tocantins, por apresentar alta produção e ser identificada entre os genótipos mais estáveis e adaptados. Destacou-se, também, a cultivar Pérola, amplamente plantada no país, que apresentou alta produção e estabilidade e adaptabilidade intermediária, pelos diferentes métodos.

Tabela 4. Estimativas de parâmetros de adaptabilidade e estabilidade fenotípica de 16 genótipos de feijoeiro-comum, avaliados em 45 ambientes na Região Central do Brasil, nos anos de 2003 e 2004, pelos métodos de Lin \& Binns (1988) modificado por Carneiro (1998), com decomposição de $\mathrm{P}_{\mathrm{i}}$ (parâmetro de estabilidade e adaptabilidade) em favorável ( $\left.\mathrm{P}_{\text {if }}\right)$ e desfavorável $\left(\mathrm{P}_{\mathrm{id}}\right)$ (Lin \& Binns modificado), trapézio quadrático ponderado pelo CV (Lin \& Binns modificado CV) e Annicchiarico (1992) (W - índice de confiança), com decomposição em ambientes favoráveis $\left(\mathrm{W}_{\mathrm{f}}\right)$ e desfavoráveis $\left(\mathrm{W}_{\mathrm{d}}\right)$.

\begin{tabular}{|c|c|c|c|c|c|c|c|c|c|c|c|c|c|c|c|c|c|c|c|}
\hline \multirow[t]{2}{*}{ Genótipo } & \multirow[t]{2}{*}{ Média $^{(1)}$} & \multicolumn{6}{|c|}{ Lin \& Binns modificado } & \multicolumn{6}{|c|}{ Lin \& Binns modificado CV } & \multicolumn{6}{|c|}{ Annicchiarico } \\
\hline & & $\begin{array}{c}\mathrm{P}_{\mathrm{i}} \\
\left(\mathrm{x} 10^{3}\right)\end{array}$ & $\mathrm{C}^{(2)}$ & $\begin{array}{c}\mathrm{P}_{\text {if }} \\
\left(\mathrm{x} 10^{3}\right)\end{array}$ & $\mathrm{C}$ & $\begin{array}{c}\mathrm{P}_{\text {id }} \\
\left(\mathrm{x} 10^{3}\right)\end{array}$ & $\mathrm{C}$ & $\begin{array}{c}\mathrm{P}_{\mathrm{i}} \\
\left(\mathrm{x} 10^{6}\right)\end{array}$ & $\mathrm{C}$ & $\begin{array}{c}\mathrm{P}_{\text {if }} \\
\left(\mathrm{x} 10^{6}\right)\end{array}$ & $\mathrm{C}$ & $\begin{array}{c}\mathrm{P}_{\mathrm{id}} \\
\left(\mathrm{x} 10^{6}\right)\end{array}$ & $\mathrm{C}$ & $\mathrm{W}_{\mathrm{i}}$ & $\mathrm{C}$ & $\mathrm{W}_{\text {if }}$ & $\mathrm{C}$ & $\mathrm{W}_{\mathrm{id}}$ & $\mathrm{C}$ \\
\hline BRS Estilo & $2.225 \mathrm{a}$ & 170 & 1 & 212 & 1 & 140 & 2 & 574 & 1 & 317 & 1 & 258 & 2 & 105,4 & 1 & 106,3 & 2 & 104,2 & 2 \\
\hline CNFC 9518 & $2.185 \mathrm{a}$ & 223 & 2 & 361 & 4 & 122 & 1 & 611 & 3 & 363 & 4 & 248 & 1 & 104,2 & 2 & 108,6 & 1 & 99,0 & 5 \\
\hline Pérola & $2.156 \mathrm{a}$ & 233 & 3 & 301 & 3 & 183 & 3 & 642 & 5 & 369 & 5 & 274 & 6 & 99,7 & 4 & 98,6 & 6 & 101,1 & 3 \\
\hline Carioca Pitoco & $2.122 b$ & 278 & 4 & 363 & 5 & 216 & 8 & 607 & 2 & 345 & 3 & 262 & 3 & 99,8 & 3 & 99,3 & 3 & 100,8 & 4 \\
\hline CNFE 8009 & $2.079 \mathrm{~b}$ & 303 & 8 & 414 & 8 & 222 & 9 & 659 & 8 & 386 & 8 & 273 & 5 & 97,1 & 6 & 96,8 & 7 & 97,9 & 7 \\
\hline IAPAR 81 & $2.075 b$ & 284 & 6 & 266 & 2 & 296 & 14 & 639 & 4 & 339 & 2 & 300 & 14 & 94,7 & 9 & 88,2 & 13 & 104,8 & 1 \\
\hline CNFC 9506 & $2.052 \mathrm{~b}$ & 298 & 7 & 418 & 9 & 210 & 5 & 653 & 6 & 385 & 7 & 268 & 4 & 97,1 & 5 & 98,8 & 5 & 95,3 & 11 \\
\hline CNFC 9458 & $2.044 \mathrm{~b}$ & 279 & 5 & 367 & 6 & 215 & 7 & 654 & 7 & 376 & 6 & 278 & 7 & 96,9 & 7 & 95,5 & 8 & 98,9 & 6 \\
\hline CNFC 9471 & $2.008 \mathrm{c}$ & 330 & 10 & 434 & 11 & 254 & 11 & 677 & 9 & 387 & 9 & 291 & 12 & 93,0 & 11 & 90,0 & 11 & 97,5 & 8 \\
\hline CNFC 9504 & $1.995 \mathrm{c}$ & 329 & 9 & 455 & 12 & 236 & 10 & 696 & 12 & 411 & 11 & 286 & 10 & 93,9 & 10 & 94,4 & 9 & 93,9 & 12 \\
\hline CNFC 9484 & $1.992 \mathrm{c}$ & 357 & 12 & 585 & 14 & 189 & 4 & 693 & 11 & 413 & 12 & 280 & 8 & 95,2 & 8 & 98,9 & 4 & 90,5 & 14 \\
\hline Magnífico & $1.963 \mathrm{c}$ & 357 & 13 & 399 & 7 & 326 & 15 & 691 & 10 & 399 & 10 & 292 & 13 & 89,8 & 14 & 86,1 & 15 & 95,4 & 10 \\
\hline CNFC 9500 & $1.961 \mathrm{c}$ & 357 & 14 & 554 & 13 & 214 & 6 & 704 & 13 & 421 & 14 & 284 & 9 & 92,5 & 12 & 94,3 & 10 & 90,6 & 13 \\
\hline CNFC 9494 & $1.961 \mathrm{c}$ & 339 & 11 & 425 & 10 & 276 & 12 & 705 & 14 & 416 & 13 & 289 & 11 & 91,7 & 13 & 88,8 & 12 & 96,2 & 9 \\
\hline BRS 9435 Cometa & $1.885 \mathrm{c}$ & 463 & 15 & 713 & 16 & 281 & 13 & 749 & 15 & 448 & 16 & 301 & 15 & 88,1 & 15 & 88,1 & 14 & 87,9 & 15 \\
\hline Carioca 11 & $1.759 \mathrm{~d}$ & 563 & 16 & 652 & 15 & 498 & 16 & 767 & 16 & 437 & 15 & 331 & 16 & 78,3 & 16 & 73,6 & 16 & 85,3 & 16 \\
\hline
\end{tabular}

${ }^{(1)}$ Médias seguidas de letras iguais não diferem entre si pelo teste Scott-Knott, a $10 \%$ de probabilidade. ${ }^{(2)}$ Classificação dos genótipos quanto à estabilidade, em cada método. 

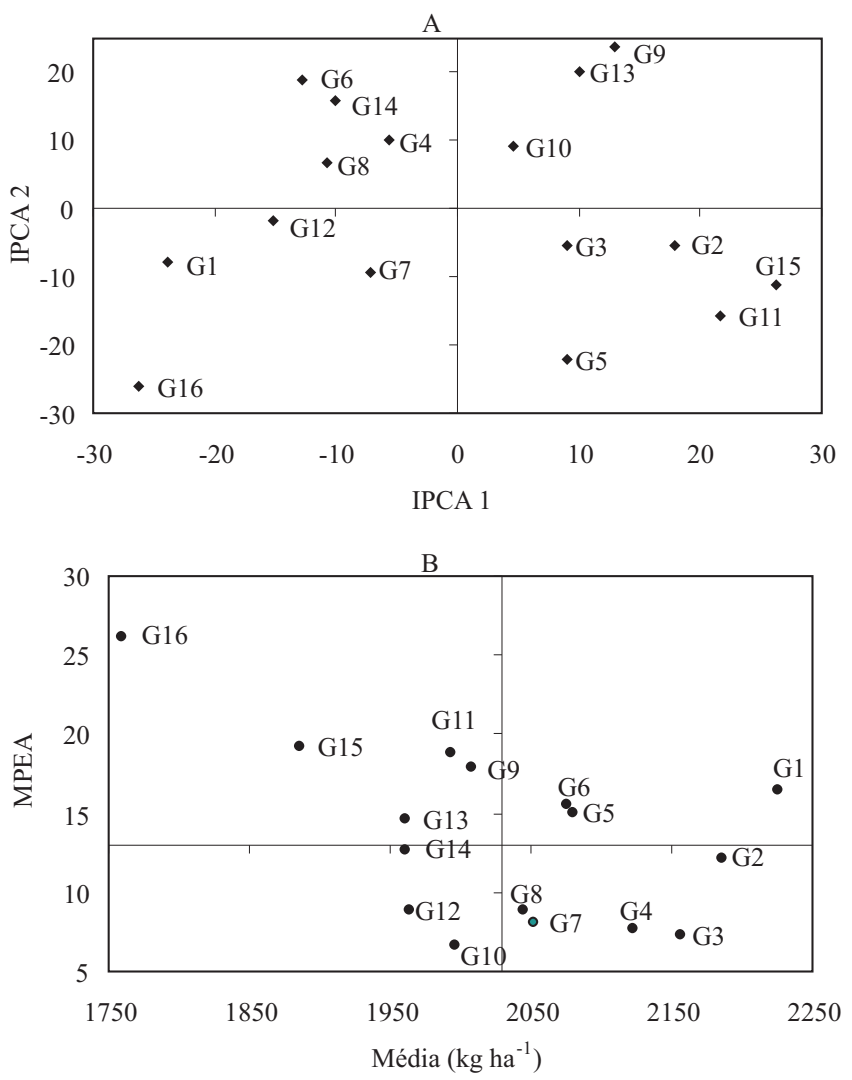

Figura 1. Análises gráficas AMMI para os 16 genótipos (G1-BRS Estilo; G2-CNFC 9518; G3-Pérola; G4-Carioca Pitoco; G5-CNFE 8009; G6-IAPAR 81; G7-CNFC 9506; G8-CNFC 9458; G9-CNFC 9471; G10-CNFC 9504; G11-CNFC 9484; G12-Magnífico; G13-CNFC 9500; G14-CNFC 9494; G15-BRS 9435 Cometa; e G16-Carioca 11), avaliados em 45 ambientes na Região Central do Brasil. A, primeiro componente principal (IPCA1) $\mathrm{x}$ segundo componente principal (IPCA2); B, média ponderada dos escores absolutos (MPEA) $\mathrm{x}$ médias de produtividade $\left(\mathrm{kg} \mathrm{ha}^{-1}\right)$.

\section{Conclusões}

1. A cultivar BRS Estilo reúne alta adaptabilidade, estabilidade e produtividade de grãos na Região Central do Brasil.

2. A cultivar Pérola ainda é boa opção para plantio na Região Central do Brasil.

\section{Referências}

ALBRECHT, J.C.; VIEIRA, E.A.; SILVA, M.S. e; ANDRADE, J.M.V de; SCHEEREN, P.L.; TRINDADE, M. da G.; SOARES
SOBRINHO, J.; SOUSA, C.N.A. de; REIS, W.P.; RIBEIRO JÚNIOR, W.Q.; FRONZA, V.; CARGNIN, A.; YAMANAKA, C.H. Adaptabilidade e estabilidade de genótipos de trigo irrigado no Cerrado do Brasil Central. Pesquisa Agropecuária Brasileira, v.42, p.1727-1734, 2007.

ALLARD, R.W.; BRADSHAW, A.D. Implications of genotype environment interactions in applied plant breeding. Crop Science, v.4, p.503-508, 1964.

ANNICCHIARICO, P. Cultivar adaptation and recommendation from alfafa trials in Northern Italy. Journal of Genetics and Plant Breeding, v.46, p.269-278, 1992.

ARIAS, E.R.A.; RAMALHO, M.A.P.; FERREIRA, D.F. Adaptabilidade e estabilidade de cultivares de milho avaliadas no Estado de Mato Grosso do Sul. Ciência e Agrotecnologia, v.20, p.415-420, 1996.

BORGES, L.C.; FERREIRA, D.F.; ABREU, A.F.B.; RAMALHO, M.A.P. Emprego de metodologias de avaliação da estabilidade fenotípica na cultura do feijoeiro-comum (Phaseolus vulgaris L.). Revista Ceres, v.47, p.89-102, 2000.

CARBONELL, S.A.M.; AZEVEDO FILHO, J.A.; DIAS, L.A. dos S.; GARCIA, A.A.F.; MORAIS, L.K. de. Common bean cultivars and line interactions with environments. Crop Breeding and Applied Biotechnology, v.61, p.169-177, 2004.

CARNEIRO, P.C.S. Novas metodologias de análise da adaptabilidade e estabilidade de comportamento. 1998. 168p. Tese (Doutorado) - Universidade Federal de Viçosa, Viçosa.

COCHRAN, W.G. The combination of estimates from different experiments. Biometrics, v.10, p.101-129, 1954.

CRUZ, C.D. Programa Genes: aplicativo computacional em genética e estatística: versão Windows. Viçosa: Editora UFV, 2001. 648p.

CRUZ, C.D.; CARNEIRO, P.C.S. Modelos biométricos aplicados ao melhoramento genético. Viçosa: Editora UFV, 2003. 579p.

CRUZ, C.D.; REGAZZI, A.J. Modelos biométricos aplicados ao melhoramento genético. Viçosa: Editora UFV, 2001. 390p.

CRUZ, C.D.; TORRES, R.A. de; VENCOVSKY, R. An alternative approach to the stability analysis proposed by Silva and Barreto. Revista Brasileira de Genética, v.12, p.567-580, 1989.

DEL PELOSO, M.J.; MELO, L.C. Potencial de rendimento da cultura do feijoeiro-comum. Santo Antônio de Goiás: Embrapa Arroz e Feijão, 2005. 131p.

EBERHART, S.A.; RUSSELL, W.A. Stability parameters for comparing varieties. Crop Science, v.6, p.36-40, 1966.

FAO. Faostat. Disponível em: <http://faostat.fao.org/site/567/ DesktopDefault.aspx?PageID=567>. Acesso em: 10 maio 2007.

FERREIRA, D.F. Aplicativo Estabilidade. Universidade Federal de Lavras. Departamento de Ciências Exatas, 2000. Disponível em: <www.ufla.br/dex>. Acesso em: 5 maio 2008.

GAUCH, H.G.; ZOBEL, R.W. AMMI analysis of yield trials. In: KANG, M.S.; GAUCH, H.G. (Ed.). Genotype by environment interaction. Boca Raton: CRC Press, 1996. p.85-122.

IBGE. Levantamento sistemático de produção agrícola. Rio de Janeiro: IBGE, 2008. 76p. 
LIN, C.S.; BINNS, M.R. A superiority measure of cultivar performance for cultivar $\mathrm{x}$ location data. Canadian Journal of Plant Science, v.68, p.193-198, 1988.

MELO, L.C.; SANTOS, P.G.; FARIA, L.C. de; DIAZ, J.L.C.; DEL PELOSO, M.J.; RAVA, C.A.; COSTA, J.G.C. da. Interação com ambientes e estabilidade de genótipos de feijoeiro-comum na Região Centro-Sul do Brasil. Pesquisa Agropecuária Brasileira, v.42, p.715-723, 2007.

OLIVEIRA, G.V.; CARNEIRO, P.C.S.; CARNEIRO, J.E. de S.; CRUZ, C.D. Adaptabilidade e estabilidade de linhagens de feijãocomum em Minas Gerais. Pesquisa Agropecuária Brasileira, v.41, p.257-265, 2006.

PIMENTEL-GOMES, F.P. Curso de estatística experimental. São Paulo: Nobel, 2000. 466p.

RAMALHO, M.A.P.; ABREU, A.F.B.; SANTOS, P.S.J. Interações genótipos $\mathrm{x}$ épocas de semeadura, anos e locais na avaliação de cultivares de feijão nas Regiões Sul e Alto Paranaíba em Minas Gerais. Ciência e Agrotecnologia, v.22, p.176-181, 1998.

ROCHA, M. de M.; FREIRE FILHO, F.R.; RIBEIRO, V.Q.; CARVALHO, H.W.L. de; BELARMINO FILHO, J.; RAPOSO, J.A.A.; ALCÂNTARA, J. dos P.; RAMOS, S.R.R.; MACHADO, C. de F. Adaptabilidade e estabilidade produtiva de genótipos de feijão-caupi de porte semi-ereto na Região Nordeste do Brasil. Pesquisa Agropecuária Brasileira, v.42, p.1283-1289, 2007.

ROCHA, M. de M.; VELLO, N.A.; LOPES, A.C. de A.; MAIA, M.C.C. Yield stability of soybean lines using additive main effects and multiplicative interaction analysis - AMMI. Crop Breeding and Applied Biotechnology, v.4, p.391-398, 2004.

ZOBEL, R.W.; WRIGHT, M.J.; GAUCH, H.G. Statistical analysis of a yield trial. Agronomy Journal, v.80, p.388-393, 1988.

Recebido em 15 de setembro de 2008 e aprovado em 15 de dezembro de 2008 